\title{
The Genitourinary Syndrome of Menopause: An Overview of the Recent Data
}

\author{
Kyveli Angelou ${ }^{1}$, Themos Grigoriadis ${ }^{1}$, Michail Diakosavvas ${ }^{1}$, Dimitris Zacharakis ${ }^{1}$, Stavros Athanasiou ${ }^{1}$ \\ 1. Urogynecology Unit, Alexandra Hospital-National and Kapodistrian University of Athens, Athens, GRC
}

Corresponding author: Michail Diakosavvas, mdiakosavvas@gmail.com

\begin{abstract}
The genitourinary syndrome of menopause (GSM) is a relatively new term for the condition previously known as vulvovaginal atrophy, atrophic vaginitis, or urogenital atrophy. The term was first introduced in 2014. GSM is a chronic, progressive, vulvovaginal, sexual, and lower urinary tract condition characterized by a broad spectrum of signs and symptoms. Most of these symptoms can be attributed to the lack of estrogen that characterizes menopause. Even though the condition mainly affects postmenopausal women, it is seen in many premenopausal women as well. The hypoestrogenic state results in hormonal and anatomical changes in the genitourinary tract, with vaginal dryness, dyspareunia, and reduced lubrication being the most prevalent and bothersome symptoms. These can have a great impact on the quality of life (QOL) of the affected women, especially those who are sexually active. The primary goal of the treatment of GSM is to achieve the relief of symptoms. First-line treatment consists of non-hormonal therapies such as lubricants and moisturizers, while hormonal therapy with local estrogen products is generally considered the "gold standard". Newer therapeutic approaches with selective estrogen receptor modulators (SERMs) or laser technologies can be employed as alternative options, but further research is required to investigate the viability and scope of their implementation in day-to-day clinical practice.
\end{abstract}

Received 03/26/2020

Review began 04/02/2020 Review ended 04/03/2020 Published 04/08/2020

() Copyright 2020

Angelou et al. This is an open access article distributed under the terms of the Creative Commons Attribution License CC-BY 4.0., which permits unrestricted use, distribution, and reproduction in any medium, provided the original author and source are credited.
Categories: Obstetrics/Gynecology, Urology

Keywords: genitourinary syndrome of menopause, vaginal atrophy, vaginal dryness, dyspareunia, estrogen replacement therapy, laser therapy

\section{Introduction And Background}

The genitourinary syndrome of menopause (GSM) is a relatively new term, first introduced in 2014 by a consensus of the International Society for the Study of Women's Sexual Health and the North American Menopause Society. GSM, previously known as vulvovaginal atrophy, atrophic vaginitis, or urogenital atrophy, is a term that describes the spectrum of changes caused by the lack of estrogens during menopause [1]. GSM-like symptoms may also be present in $15 \%$ of premenopausal women due to the hypoestrogenic state [2]. Nonetheless, the vast majority of women suffering from GSM are of older age, with $50-70 \%$ of postmenopausal women being symptomatic at least to some degree [3]. To this day, GSM remains extremely underdiagnosed despite its high prevalence, mostly because of the reluctance among women to seek help due to embarrassment, or as a result of a tendency among many women to consider it as a normal feature of natural aging. However, in many cases, the reluctance of healthcare professionals to address these issues constitutes a major cause of the lack of awareness about this syndrome among affected women $[4,5]$.

\section{Review}

\section{Clinical manifestations and evaluation}

GSM is a chronic, progressive condition of the vulvovaginal and lower urinary tract, which is characterized by a broad spectrum of signs and symptoms. The common clinical manifestations of the condition are summarized in Table 1. 


\section{Cureus}

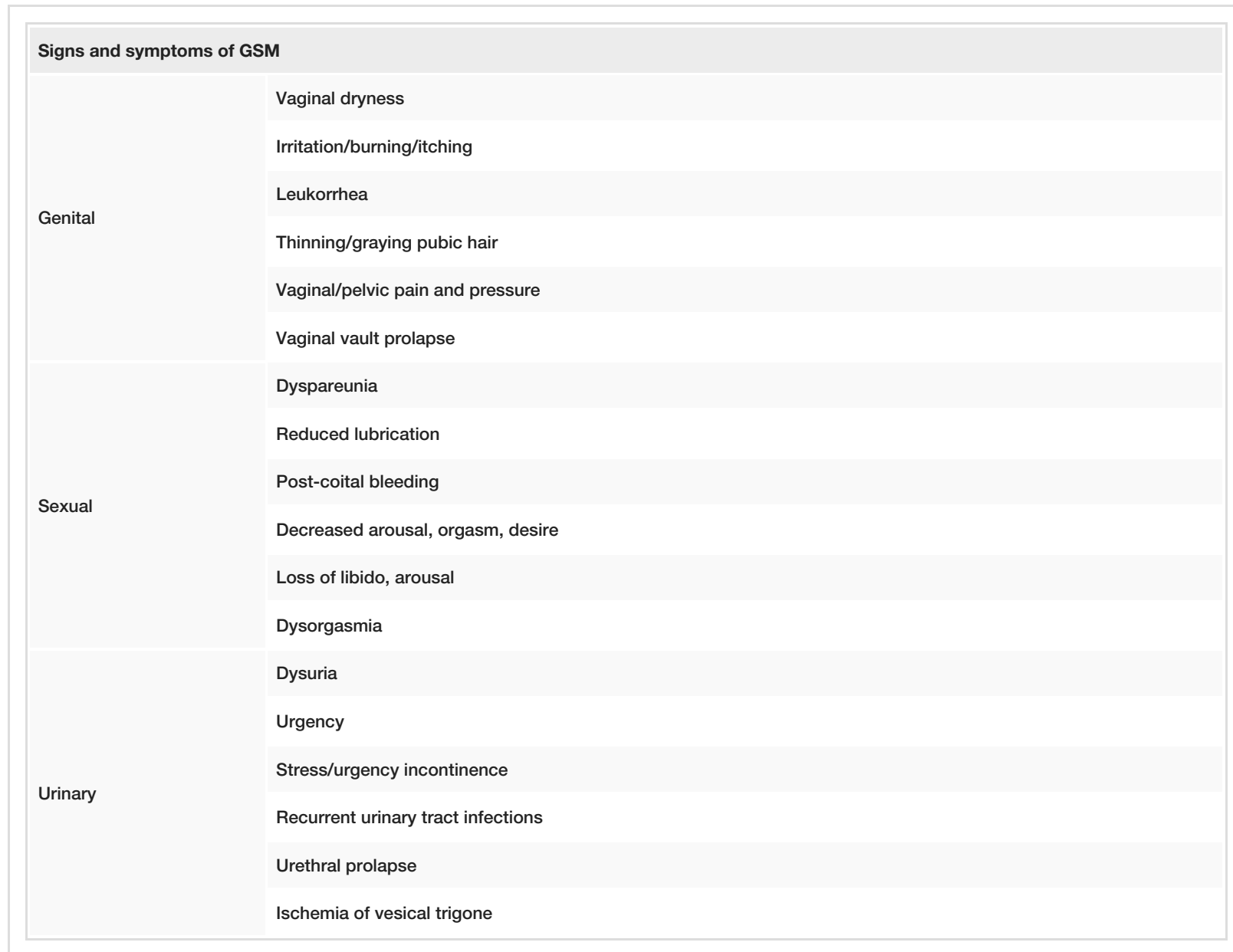

TABLE 1: Major clinical manifestations of GSM

GSM: genitourinary syndrome of menopause

The diagnosis of GSM may prove to be challenging as the clinical manifestations of GSM are mild and nonspecific in approximately $50 \%$ of postmenopausal women [2]. An observational study by Moral et al. found that vaginal dryness is the most prevalent and bothersome symptom as it affects up to $93 \%$ of women; the study also noted that this symptom is characterized as being moderate to severe in intensity in $68 \%$ of the cases [3]. Irritation and burning/itching of vulva/vagina are other symptoms that women with GSM frequently complain about, and they are reported in $63.3 \%$ of the affected women. The most predominant complaints of sexually active women are reduced lubrication and dyspareunia, the prevalence of which has been reported to be $90 \%$ and $80 \%$ respectively. Loss of libido and arousal and per vagina bleeding or spotting during or after intercourse are also frequently reported. Urinary symptoms are considered less frequent with dysuria (29\%), urgency and urge incontinence (28\%), recurrent urinary tract infections, stress incontinence, and voiding issues being some of the most common manifestations [3,6]. Moreover, other common signs of GSM include decreased moisture (94\%), loss of vaginal rugae (78\%), vaginal pallor (75\%), and decreased elasticity (68\%). Finally, pelvic organ prolapse, such as cystocele, rectocele, prolapse of the uterus, or vaginal vault prolapse, is also related to GSM [1,2,7].

The prevalence and severity of the above-mentioned symptoms vary in relation to time passed since menopause, with most of them being more frequent and intense five years after menopause when compared with women closer to premenopausal status (GSM symptoms occur in $84 \%$ of women six years after menopause versus one year postmenopausally in $65 \%$ ) $[3,8]$. Contrary to the vasomotor symptoms related to menopause, which tend to be milder over time, symptoms of GSM appear to have a greater impact on the quality of life (QOL) of the affected women. This is due to the fact that they rarely resolve spontaneously and, in most cases, deteriorate if left untreated, thereby negatively affecting patients' confidence and intimacy with their partners $[9,10]$. The impact in the QOL has been shown to be more profound in sexually active women [3].

The diagnosis and evaluation of GSM are clinical and mostly established through a thorough medical history and physical pelvic examination [11]. Women who are peri- or postmenopausal or with other causes of 


\section{Cureus}

hypoestrogenism should be evaluated for signs and symptoms of urogenital atrophy during routine clinical visits $[10,11]$. Laboratory testing is not typically required, although cultures or biopsies could be indicated if appearance is non-typical or does not improve after therapy [12]. Some tests to assess the efficacy of therapies such as the vaginal $\mathrm{pH}$ and the vaginal maturation index (VMI) are mainly used in research studies and are not considered essential for the diagnosis of GSM. Clinicians should always exclude other causes with similar symptoms and, specifically, dermatological conditions of the vulva such as lichen sclerosus or planus, eczema, dermatitis, chronic vulvovaginitis, vaginitis and vaginosis, vulvodynia, malignancies, and chronic pelvic pain [13]. Recently, two instruments of measurement properties of patient-reported outcome measures (PROMs) specific for genitourinary symptoms (the Vulvovaginal Symptoms Questionnaire and the Day-to-Day Impact of Vaginal Aging (DIVA) Questionnaire) have been shown to be valid after a thorough assessment. It has been shown these two tools can be efficiently used for the evaluation of GSM symptoms and measurement of their impact on the QOL of patients $[14,15]$.

\section{Pathophysiology and risk factors}

Most of the symptoms of GSM are attributed to the lack of estrogen that characterizes menopause. The anatomical and pathophysiological changes caused by GSM are presented in Table 2.

Pathophysiology of GSM

Loss of labial and vulval thickness

Decreased collagen, elasticity, and blood flow

Reduced vaginal discharge

Reduced pubic hair, subcutaneous fat of labia majora

Reduced labia minora and hymenal remnants

Decreased vaginal cells glucogen $=>$ change vaginal microbiome $=>$ increased $\mathrm{pH}$

Decreased pelvic floor strength and control

Dry and thin epithelium

Short and narrow vagina

Prolapse (vaginal vault, pelvic organs, urethral)

Decreased bladder capacity and sensation

Vaginal hypersensitivity or decreased feeling

TABLE 2: Anatomical and pathophysiological changes caused by GSM

GSM: genitourinary syndrome of menopause

There are three types of endogenous estrogens (estradiol, estrone, and estriol) with varying ratios as age progresses. Estradiol prevails in premenopausal women, whereas estrone, a less effective form, is the predominant estrogen in menopause, explaining the hypoestrogenic environment in these ages $[2,11]$. The common embryonic origin during fetal development of the lower urinary tract and external genitalia explains the pathophysiologic effects of the lack of estrogens on these anatomical structures. It has been shown that estrogen receptors ( $a$ and $b$ ) are present in the vagina, the vestibule of the vulva, urethra, trigone of the bladder, and on autonomic and sensory neurons in the vagina and vulva. While both receptors can be found in premenopausal women, only estrogen-b receptors can be detected in postmenopausal women [12]. Irritation and trauma occurring during sexual intercourse is the result of the hypoestrogenic environment of urogenital tract tissue, and It reflects changes in the thickness of the vaginal epithelium and lamina propria, atrophy of smooth muscles, reduction of vaginal-area blood flow, and loss of tissue elasticity (decreased concentration of collagen, elastin, and hyaluronic acid) [5,6]. Diminution of glycogen concentration in vaginal cells results in an alteration in the vaginal microbiome (fewer lactobacilli) and an increase of vaginal $\mathrm{pH}$ [1]. The combined effect of these pathophysiological mechanisms results in changes of the urogenital system, mainly as alterations of the vaginal mucosa (pallor and fragility) and discharge, changes in vaginal microbe consistency, a decrease of pubic hair and subcutaneous fat of labia majora, and loss of size of labia minora and vestibular bulbs. In the lower urinary tract, they affect the capacity and contractile ability of the bladder, the urethral sphincter, and the functionality of pelvic floor muscles [16]. 


\section{Cureus}

The main risk factors recognized to be associated with GSM besides the menopause itself are the absence of vaginal childbirth, alcohol abuse, bilateral oophorectomy, decreased frequency and sexual abstinence, cigarette smoking, non-menopausal hypoestrogenism, lack of exercise, (premature) ovarian failure, and cancer treatments via pelvic irradiation, chemotherapeutic, and endocrinal agents $[4,6,17]$. Similarly, low education levels and chronic diseases, mostly urogynecological pathologies, have also been associated with GSM, while BMI has not been correlated with GSM onset [18].

\section{Therapeutic approach}

The primary goal of GSM's treatment is symptom relief. The available treatment options, besides local and systemic hormonal therapy, include lifestyle changes and non-hormonal treatments (Table 3) [5,19,20].

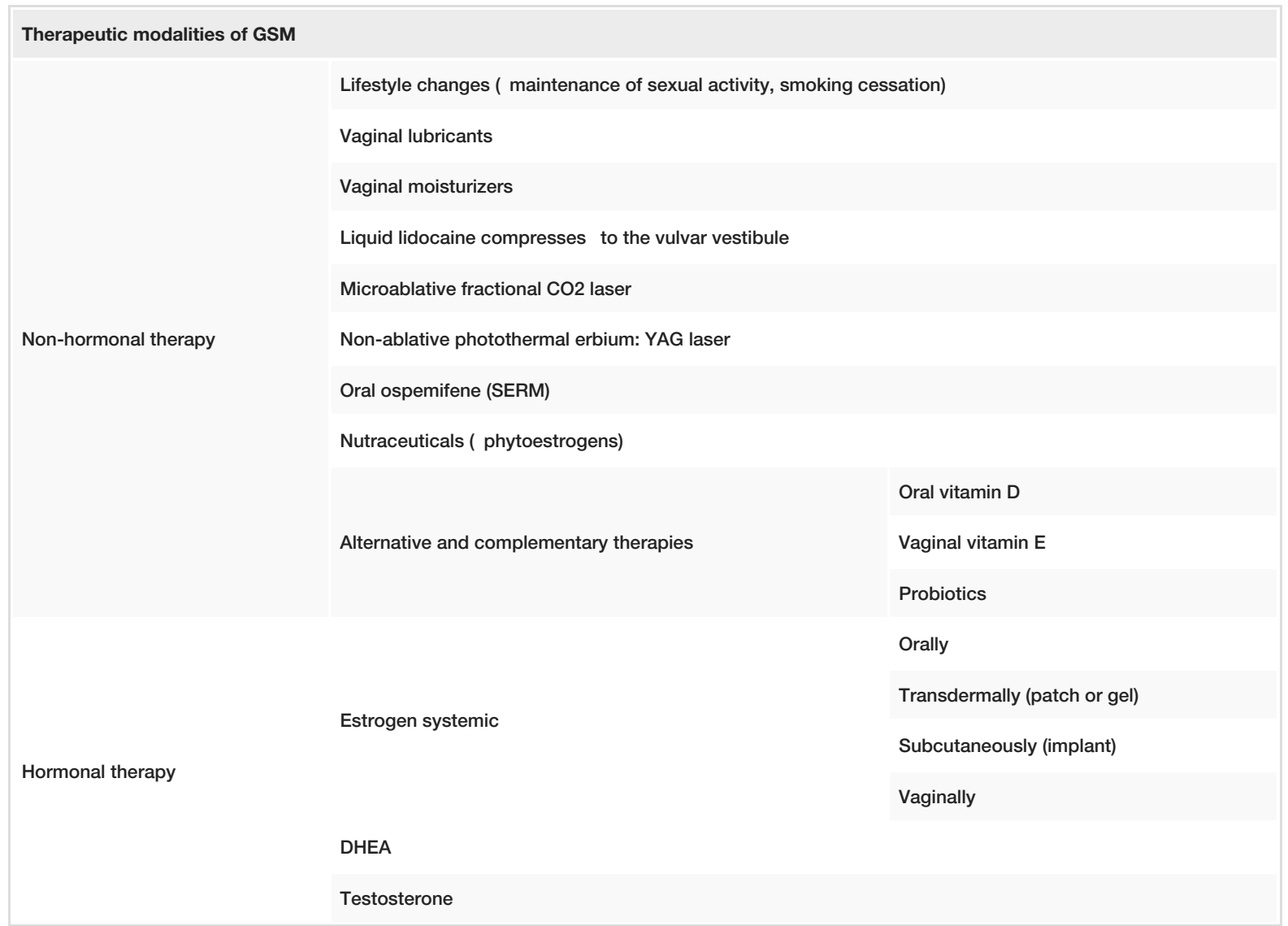

\section{TABLE 3: Treatment options for GSM}

GSM: genitourinary syndrome of menopause; SERM: selective estrogen receptor modulator; DHEA: dehydroepiandrosterone

Concerning the conservative approach, a positive link between maintenance of vaginal elasticity and lubricative response to sexual arousal with sexual activity has been demonstrated [21]. Moreover, avoiding any risk factors associated with GSM, such as smoking cessation, can be helpful, since smoking has been linked with an increase in estrogen metabolism leading to vaginal atrophy [19]. Nevertheless, the first-line treatment for GSM is the use of non-hormonal vaginal lubricants and moisturizers [22]. Lubricants are water-, silicone-, or oil-based products that are not skin absorbed. They act immediately and provide temporary relief from vaginal dryness and pain during sex; hence, they are really helpful for women whose vaginal dryness is an issue only or primarily during intercourse. On the other hand, vaginal moisturizers are applied regularly. They are bioadhesives and can improve coital comfort and increase vaginal moisture. Moisturizers mimic vaginal secretions and lower the $\mathrm{pH}$ by altering the fluid content in the vaginal epithelium. However, both these products are appropriate mostly for women with mild to moderate symptoms, and many patients will eventually require further hormonal medications [6,23]. Additionally, the use of liquid lidocaine compresses to the vulvar vestibule before sexual penetration has been described in breast cancer survivors with menopausal dyspareunia who should not receive estrogen-based therapy. This has shown promising results since $90 \%$ of patients reported comfortable intercourse [24]. Other complementary therapies such as oral vitamin $\mathrm{D}$, vaginal vitamin $\mathrm{E}$, and probiotics have been proposed as alternative modalities to GSM therapy; however, data on their efficacy are scarce and further studies and 
trials are required for their validation [25-27]. More recently, phytoestrogens, specifically equol, have been proven effective in modulating symptoms of menopause. However, due to insufficient research and limited data in the literature, they cannot yet be recommended as an alternative treatment option [28].

In more persistent cases, hormonal therapy with estrogen products is generally considered the "gold standard". The use of hormonal therapy is an option for the treatment of moderate to severe GSM symptoms that are not relieved with conservative methods. It may also be used in conjunction with a conservative approach $[2,7]$. The hormonal therapy includes estrogen-based products that can be administered through different routes (vaginally, orally, transdermally or subcutaneously), with vaginal tablets or creams showing better outcomes than the others [5]. Estrogens delivered orally, in many cases, do not lead to beneficial effects on the lower genital tract. Locally administered Intravaginal estrogen options (tablets, rings, creams) with low estradiol concentration ( $\leqslant 50 \mathrm{mcg}$ estradiol or $50 \mathrm{mcg}$ estriol) have shown good outcomes in treating GSM symptoms such as dryness, itchiness, vaginal mucosa friability, and dyspareunia [11]. Concerning urinary tract symptoms, improvement has been noticed in many cases of recurrent urinary tract infections and overactive bladder (without any sign of microbial involvement), but not in women with stress incontinence. In cases with a history of estrogen-sensitive tumors, such as breast or endometrial cancer, any hormonal therapy should be used with caution; the risk/benefit ratio needs to be individualized and in coordination with an oncologist [19]. Hormonal therapy risks should be evaluated thoroughly and factors such as age, duration of use, dose, type of treatment, route, histologic type of malignancy, and prior exposure should be considered before the prescription of such regimen in survivors of gynecological cancer [29]. Breast cancer is a hormone-sensitive carcinoma in many cases; hence, systemic hormonal therapy is not usually recommended for women with breast cancer [29,30]. As far as endometrial cancer survivors are concerned, the data, although limited, suggest that hormonal therapy is considered relatively safe in low-risk subtypes (i.e., early-stage, low-grade, or type I), but should not be employed in high-risk types [29,31]. Similarly, in survivors of epithelial ovarian cancer, hormonal treatment could be considered in selected cases, since evidence suggests a neutral effect in survival; but it should be avoided in certain histologic types, such as advanced serous and endometrioid and other estrogen-sensitive tumor types [29-31].

Another medication used to treat GSM symptoms is the intravaginal dehydroepiandrosterone (DHEA). Studies have shown that DHEA, increases blood estrogen concentration, thereby leading to the improvement of sexual arousal and the return of lost libido [32]. Intravaginal testosterone has shown positive effects in relieving vaginal atrophy symptoms and decreased libido, but its efficacy in GSM generally remains uncertain [33]. Another available option is an oral selective estrogen receptor (SERM) known as ospemifene. It is used mainly for the treatment of dyspareunia and is employed in cases of women who cannot be treated with conventional and medical products due to estrogen contradictions or those who have not responded to other therapies. However, the efficacy of SERMs as a treatment option for GSM needs to be further investigated [34].

Recently, laser and radiofrequency devices have emerged as alternative treatment modalities for GSM [35]. It is suggested that microablative fractional $\mathrm{CO} 2$ and non-ablative erbium: YAG (Er:YAG) lasers restore the tropism in the lower genitourinary tract with three to five sessions [35,36]. Despite the initial excitement for this new therapeutic approach, it has not been fully implemented in the day-to-day practice to this day, and its routine use is not recommended by some scientific societies [20]. Nonetheless, a recent study has reported that laser intervention with the intravaginal use of either $\mathrm{CO} 2$ or Er:YAG laser-technologies is a safe and potentially effective nonpharmacologic intervention for GSM [37].

\section{Conclusions}

GSM results from the hypoestrogenic state that accompanies menopause, and it affects most of the postmenopausal women. Due to different hormonal and anatomical changes, GSM is characterized by various symptoms such as vaginal dryness, dyspareunia, and dysuria, thereby leading to a drastic impact on the QOL of affected women. Recently, different treatment modalities have emerged to treat the condition's bothersome and life-changing symptoms. First-line treatment consists of non-hormonal therapies such as lifestyle changes, lubricants, and moisturizers, while hormonal therapy with locally administered intravaginal estrogen products is considered the "gold standard" in more persistent cases. Newer therapeutic approaches with SERMs or laser technologies can be employed as alternative options, but further research is required to analyze their implementation in day-to-day clinical practice.

\section{Additional Information}

\section{Disclosures}

Conflicts of interest: In compliance with the ICMJE uniform disclosure form, all authors declare the following: Payment/services info: All authors have declared that no financial support was received from any organization for the submitted work. Financial relationships: All authors have declared that they have no financial relationships at present or within the previous three years with any organizations that might have an interest in the submitted work. Other relationships: All authors have declared that there are no other relationships or activities that could appear to have influenced the submitted work. 


\section{References}

1. Portman DJ, Gass ML: Genitourinary syndrome of menopause: new terminology for vulvovaginal atrophy from the International Society for the Study of Women's Sexual Health and the North American Menopause Society. Menopause. 2014, 21:1063-1068. 10.1097/GME.0000000000000329

2. Gandhi J, Chen A, Dagur G, Suh Y, Smith N, Cali B, Khan SA: Genitourinary syndrome of menopause: an overview of clinical manifestations, pathophysiology, etiology, evaluation, and management. Am J Obstet Gynecol. 2016, 215:704-711. 10.1016/j.ajog.2016.07.045

3. Moral E, Delgado JL, Carmona F, et al.: Genitourinary syndrome of menopause. Prevalence and quality of life in Spanish postmenopausal women. The GENISSE study. Climacteric. 2018, 21:167-173. 10.1080/13697137.2017.1421921

4. Mac Bride MB, Rhodes DJ, Shuster LT: Vulvovaginal atrophy. Mayo Clin Proc. 2010, 85:87-94. 10.4065/mcp.2009.0413

5. Briggs P: Genitourinary syndrome of menopause. Post Reprod Health. 2019, 2053369119884144. $10.1177 / 2053369119884144$

6. Farrell Am E: Genitourinary syndrome of menopause. Aust Fam Physician. 2017, 46:481-484.

7. Kim H, Kang SY, Chung YJ, Kim JH, Kim MR: The recent review of the genitourinary syndrome of menopause. J Menopausal Med. 2015, 21:65-71. 10.6118/jmm.2015.21.2.65

8. Palma F, Volpe A, Villa P, Cagnacci A: Vaginal atrophy of women in postmenopause. Results from a multicentric observational study: The AGATA study. Maturitas. 2016, 83:40-44. 10.1016/i.maturitas.2015.09.001

9. Sturdee DW, Panay N: Recommendations for the management of postmenopausal vaginal atrophy . Climacteric. 2010, 13:509-522. 10.3109/13697137.2010.522875

10. Nappi RE, Kokot-Kierepa M: Vaginal Health: Insights, Views \& Attitudes (VIVA) - results from an international survey. Climacteric. 2012, 15:36-44. 10.3109/13697137.2011.647840

11. Phillips NA, Bachmann GA: Genitourinary syndrome of menopause: common problem, effective treatments Cleve Clin J Med. 2018, 85:390-398. 10.3949/ccjm.85a.15081

12. Shifren JL: Genitourinary syndrome of menopause. Clin Obstet Gynecol. 2018, 61:508-516. 10.1097/GRF.0000000000000380

13. The North American Menopause Society: The role of local vaginal estrogen for treatment of vaginal atrophy in postmenopausal women: 2007 position statement of the North American Menopause Society. Menopause. 2007, 14:355-369. 10.1097/gme.0b013e31805170eb

14. Gabes M, Knüttel H, Stute P, Apfelbacher CJ: Measurement properties of patient-reported outcome measures (PROMs) for women with genitourinary syndrome of menopause: a systematic review. Menopause. 2019, 1342-1353. 10.1097/GME.0000000000001390

15. Erekson EA, Yip SO, Wedderburn TS, et al.: The Vulvovaginal Symptoms Questionnaire: a questionnaire for measuring vulvovaginal symptoms in postmenopausal women. Menopause. 2013, 20:973-979. 10.1097/GME.0b013e318282600b

16. Mitchell CM, Waetjen LE: Genitourinary changes with aging. Obstet Gynecol Clin North Am. 2018, 45:737750. 10.1016/j.ogc.2018.07.010

17. Goldstein I: Recognizing and treating urogenital atrophy in postmenopausal women. J Womens Health (Larchmt). 2010, 19:425-432. 10.1089/jwh.2009.1384

18. Karakoç H, Uçtu AK, Özerdoğan N: Genitourinary syndrome of menopause: effects on related factors, quality of life, and self-care power. Prz Menopauzalny. 2019, 18:15-22. 10.5114/pm.2019.84152

19. Palacios S, Mejía A, Neyro JL: Treatment of the genitourinary syndrome of menopause . Climacteric. 2015, 18:23-29. 10.3109/13697137.2015.1079100

20. Palacios S, Combalia J, Emsellem C, Gaslain Y, Khorsandi D: Therapies for the management of genitourinary syndrome of menopause. Post Reprod Health. 2020, 26:32-42. 10.1177/2053369119866341

21. Sánchez-Borrego R, Manubens M, Navarro MC, et al.: Position of the Spanish Menopause Society regarding vaginal health care in postmenopausal women. Maturitas. 2014, 78:146-150. 10.1016/j.maturitas.2014.03.003

22. American College of Obstetricians and Gynecologists: ACOG Practice Bulletin No. 141: management of menopausal symptoms. Obstet Gynecol. 2014, 123:202-216. 10.1097/01.AOG.0000441353.20693.78

23. The North American Menopause Society: Management of symptomatic vulvovaginal atrophy: 2013 position statement of the North American Menopause Society. Menopause. 2013, 20:888-902. 10.1097/GME.0b013e3182a122c2

24. Goetsch MF, Lim JY, Caughey AB: A practical solution for dyspareunia in breast cancer survivors: a randomized controlled trial. J Clin Oncol. 2015, 33:3394-3400. 10.1200/JCO.2014.60.7366

25. Yildirim B, Kaleli B, Düzcan E, Topuz O: The effects of postmenopausal vitamin D treatment on vaginal atrophy. Maturitas. 2004, 49:334-337. 10.1016/j.maturitas.2004.02.008

26. Costantino D, Guaraldi C: Effectiveness and safety of vaginal suppositories for the treatment of the vaginal atrophy in postmenopausal women: an open, non-controlled clinical trial. Eur Rev Med Pharmacol Sci. 2008, 12:411-416.

27. Muhleisen AL, Herbst-Kralovetz MM: Menopause and the vaginal microbiome. Maturitas. 2016, 91:42-50. 10.1016/j.maturitas.2016.05.015

28. Caruso S, Cianci S, Fava V, Rapisarda AMC, Cutello S, Cianci A: Vaginal health of postmenopausal women on nutraceutical containing equol. Menopause. 2018, 25:430-435. 10.1097/GME.0000000000001061

29. Harris BS, Bishop KC, Kuller JA, Ford AC, Muasher LC, Cantrell SE, Price TM: Hormonal management of menopausal symptoms in women with a history of gynecologic malignancy. Menopause. 2020, 27:243-248. 10.1097/GME.0000000000001447

30. Temkin SM, Mallen A, Bellavance E, Rubinsak L, Wenham RM: The role of menopausal hormone therapy in women with or at risk of ovarian and breast cancers: misconceptions and current directions. Cancer. 2019, 125:499-514. 10.1002/cncr.31911

31. Kapoor E, Benrubi D, Faubion SS: Menopausal hormone therapy in gynecologic cancer survivors: a review of 


\section{Cureus}

the evidence and practice recommendations. Clin Obstet Gynecol. 2018, 61:488-495.

10.1097/GRF.0000000000000381

32. Martel C, Labrie F, Archer DF, et al.: Serum steroid concentrations remain within normal postmenopausal values in women receiving daily 6.5mg intravaginal prasterone for 12 weeks. J Steroid Biochem Mol Biol. 2016, 159:142-153. 10.1016/j.jsbmb.2016.03.016

33. Witherby S, Johnson J, Demers L, et al.: Topical testosterone for breast cancer patients with vaginal atrophy related to aromatase inhibitors: a phase I/II study. Oncologist. 2011, 16:424-431.

10.1634/theoncologist.2010-0435

34. Szymański JK, Siekierski BP, Kajdy A, Jakiel G: Post-menopausal vulvovaginal atrophy - an overview of the current treatment options. Ginekol Pol. 2018, 89:40-47. 10.5603/GP.a2018.0008

35. Athanasiou S, Pitsouni E, Falagas ME, Salvatore S, Grigoriadis T: CO2-laser for the genitourinary syndrome of menopause. How many laser sessions?. Maturitas. 2017, 104:24-28. 10.1016/j.maturitas.2017.07.007

36. Salvatore S, Pitsouni E, Del Deo F, Parma M, Athanasiou S, Candiani M: Sexual function in women suffering from genitourinary syndrome of menopause treated with fractionated CO2 laser. Sex Med Rev. 2017, 5:486494. 10.1016/j.sxmr.2017.07.003

37. Pitsouni E, Grigoriadis T, Falagas ME, Salvatore S, Athanasiou S: Laser therapy for the genitourinary syndrome of menopause. A systematic review and meta-analysis. Maturitas. 2017, 103:78-88.

10.1016/j.maturitas.2017.06.029 